\title{
The Difference Between Using Proxy Server and VPN
}

\author{
David Dwiputra Kurniadi \\ Faculty of Computer Science Department of Information System \\ Pawiyatan Luhur Street IV / 1 \\ daviddwiputra79@yahoo.com
}

\begin{abstract}
In this era of globalization, it is important to get the lastest information or update. In this time people must know what internet is as almost every person ing computer or gadget, using internet to search for some information or something new.

For example, looking for software, game through internet. But sometimes, there are some websites that cannot be opened as they have Internet Positive notificatio. To solve that problem, hacker found the solution by creating Proxy Server or VPN. In this time internet is very modern and very easy to access and there are a lot of Proxy Server and VPN that can be easly used.
\end{abstract}

Keywords : Proxy, Proxy Server, Web Server, Internet, Virtual Private Network, VPN

\section{Introduction}

In this time technology is very modern, especially the development of internet technology. Moreover, now internet can be used anywhere, at home or in the cafe. And internet can be used to contact each other or interchange news with other people. Now, looking for some information is very easy.

Just open the internet and people can get the lastest information or uptodate information. Internet can be also be used for everybody form diffrent age, even toddlers can use internet. But there are some blocked website and websites that are very slow to open / access. Because there are some problems that make certain websites cannot be opened or accessed, make a server slow, the writer will disscuss about Proxy Server and VPN.

\section{Literature Review}

The writer will disscuss Proxy Server and VPN, but first the writer will explain what internet is. Internet is an abbreviation of Interconnected-Networking. Internet is an international network for computer created by the United States in 1969. At the begining the United States created internet only for military needs; it was, used to solve the problems that might arise if there was a nuclear attack.

It means that the military was afraid if their base was hit by a nuclear attack, they would lose important data from certain machines because the machines broke. Because of that the United States created an internet, so if the main engine broke down, the important data is not gone.

But in this era, internet technology is more developed and internet speed becomes faster, so that the internet can be used by everybody and can be accessed easily. Now, more than 160 countries know internet and use internet. The requirements are, the user must have gadget and connection to use internet. But only with internet, browsing itself is less comfortable, because there are some blocked websites.

Because of that the writer will explain about Proxy Server and VPN, because that thing are very important for internet user. Moreover, proxy dan VPN can unblock the blocked website and also can increase the browsing speed. Even Proxy Server is integrated with Firewall Server, although both of them work at different 
layer. Firewall functions to protect the lokal network from the interference that comes from the outside network, while proxy server work at application layer.Usually Firewall already adds on routers to filter a package that passes from connected networks. Now, we go to Proxy Server and VPN disscussion.

\subsection{Proxy Server}

Proxy Server can be understood as a third party that stands between two parties connected to each other and functions as a middleman. So, the first party and second party are connected indirectly by, the middleman, called proxy. The first party is Client Application and the second party is Server Application. Client Application and Server Application are not only one, but there are many. For example Client Application are web browser, FTP, etc, and Server Application are web server, FTP, etc. What is Proxy Server and Web Proxy?

Proxy Server is a server that can be used to handle or monitor a traffic data that are still operating.

\subsection{Web Proxy}

Web Proxy is a website that hides IP Address, because of that user IP Address cannot be detected because the IP is already hidden by the proxy. Web Proxy also has another function, like saving bandwith and increasing browsing speed.

There are two types of Web Proxy, they are:

1. Nontransparent Web Proxy

2. Transparent Web Proxy

Nontransparent Web Proxy does not increase internet speed, it even decreases the internet speed. But Nontransparent Web Proxy can block or hide the user IP Address, so that the IP Address user cannot be detected by a server.

Transparent Web Proxy can increase the speed to access some websites, but Transparent Web Proxy cannot hide the user IP Address, so user IP Address can be detected by the server.

\subsection{Proxy Server Functions}

Proxy Server have three main functions,they are :

1. Connection sharing

2. Filtering

3. Caching

Connection Sharing is a gateway that be a barrier between lokal network with outside network.Gateway is also used as a point where the number of connections from local user and outside user are connected.Because of that the connection from the local network to internet will use gateway connection collectively

Filtering functions as Firewall to protect local network from the outside network interference.

Caching is a place to save requested objects from the users. If there is a site / website that has already been registered, the user can access it faster.

\subsection{Virtual Private Network}

VPN is the abbreviation of Virtual Private Network. VPN is a connection between one network with another network privately. Because the connection is private,the data the user sends will be kept safe and secret although they are sent through public connection.

To use VPN, the step is very easy, just open an existing VPN website. For example, Vpnbook, SurfEasy VPN,etc. 


\subsection{Benefits of Virtual Private Network}

VPN have three main functions, they are :

1. Remote Access

2. Security

3. Saving cost of network setup

Remote Access means users can access the computer network or gadget anywhere as long as they are connected to internet.

Security means users can use internet freely or can use hotspot from the restaurants safely.

VPN can save the cost of network setup because the data transmission used by VPN is using the existing internet network media, so there is no need to build one's own network.

\subsection{Advantages and Disadvantages of VPN}

Advantages :

1. Hide IP Address

2. Unblock the Blocked site/website

3. Easy to change IP

4. Increase the internet speed

Disadvantages :

1. Internet connection cannot be predicted

2. It is vulnerable to be hacked, because VPN works in public channel

3. It requires cost, because some VPNs require transaction to become a member

\subsection{Advantages and Disadvantages of Proxy Server}

Advantages :
1. Local network is safe ( secure ) from outside interference

2. Increase browsing speed

3. Unblock the blocked site / website

4. Save bandwith

5. Hide user IP Address

Disadvantages :

1. Access to a site/website that has not been accessed before is slow, because the client must register first to the proxy

2. User will see an expired file if the cache expire time is too long

3. When connected to slow IP, user must look for another IP, which is wasting time

4. If there are many users using the same proxy server, it will slow down the proxy performance and slow down the internet speed

\section{Research Methodology}

To search more about the difference between Proxy Server and VPN, the writer used observation method, because according to the writer observation method can save time, no need to ask people or ask people to fill the questionnaire.

Observation that writer did are comparing between Proxy Server and VPN.

\section{Result and Discussion}

The things that the writer obtained from the observation was that the writer did internet speed test of Proxy and VPN, proved the speed by opening a blocked website/site, and hide the IP Address.

The result of the internet speed observation that the writer did in speedtest.net website shown that by, using proxy the speed of download was $6.64 \mathrm{Mbps}$ and the speed of upload was $18.08 \mathrm{Mbps}$, on the other hand by using VPN the download speed was 6.93Mbps and the upload speed was 19.02 Mbps. The VPN that the writer used was Vpnbook.com.

To prove that blocked website can be accessed with proxy and VPN, the writer used vimeo.com website. At first vimeo.com 
could not be accessed with Internet Positive Notification, but by using proxy and VPN, vimeo.com could be accessed.To access using proxy, the writer opened the browser settings first and inputted the IP Address and also inputted the Port that writer got from hidemyass.com. And to access website/site using VPN the writer just opened Vpnbook.com and inputted vimeo.com at Free Web table.

Actually proxy dan VPN do not hide the user IP Address,but the user uses the IP from the outside and uses it on VPN server.

\section{Conclusion}

The development of technology is very fast. First, internet can be used for military needs. Because techonolgy always evolves and internet speed is faster, now internet can be used by everyone. But using internet is not free,to use internet user must have a gadget and internet connection.

It is not free but with minimum cost, user can access the internet.

With internet,user can searchany information easily,but internet is not enough,because there are a lot of blocked website / site,and also slow internet speed to access some websites / sites. Because of that a proxy or VPN is created to unblock the blocked site/website,and also increases speed to access some websites / sites. Another thing is proxy and VPN can hide user IPAddress.

But using proxy or VPN is relative, depends on the IP that user uses. But the point is VPN is better than proxy,because VPN increases internet speed better than proxy, and also using VPN is easier then using proxy. Moreover, by using VPN, all online activity cannot be seen by ISP ( Internet Service Provider )

\section{References}

[1]http://www.kajianpustaka.com/201 3/09/pengertian-manfaat-dan-fungsiproxy.html

[2]http://www.webopedia.com/TERM $\underline{\text { I/Internet.html }}$

[3]http://www.scribd.com/doc/14451 010/contoh-

Acknowledgement\#scribd

[4] https://gerakanopensource.wordpres s.com/2011/09/13/pengertian-proxy/

\{5]http://www.transiskom.com/2014/10 Lpengertian-vpn-virtual-privatenetwork.html

[6]http://bisnis.tempo.co/read/news/20 15/03/31/090654239/ini-daftar-19situs-yang-diblokir-kominfo 